\title{
Whole genome of novel Lactobacillus fermentum HFD1 strain producing various antimicrobial metabolites
}

\author{
Georgii Ozhegov \\ Kazan Federal University, \\ Kazan, Russia \\ Perm State Pharmaceutical Academy, \\ Perm, Russia \\ georgii_provisor@mail.ru \\ Alexey Vasilchenko \\ University of Tyumen, \\ Tyumen, Russia
}

\author{
Monyr Nait Yahia \\ Kazan Federal University, \\ Kazan, Russia
}

\author{
Natalya Gogoleva \\ Kazan Federal University, \\ Kazan Institute of Biochemistry and \\ Biophysics, FRC Kazan Scientific \\ Center of RAS, Kazan, Russia
}

\author{
Dina Yarullina \\ Kazan Federal University, \\ Kazan, Russia
}

\author{
Airat Kaumov \\ Kazan Federal University, \\ Kazan, Russia \\ kairatr@yandex.ru
}

\begin{abstract}
Lactobacilli, generally recognized as safe for humans, are characterized with high antagonistic properties. Among 40 strains of Lactobacilli isolated from the faeces of healthy humans, Lactobacillus fermentum HFD1 exhibited significant antimicrobial activity against various nosocomial pathogens. The genome of $L$. fermentum HFD1 was sequenced on Illumina MiSeq and ONT MinION instruments. After reads assembly 2 circular contigs with respective sizes of 2101878 bp and 5386 bp have been obtained. The raw reads alignment with BWA and post-assembly assessment showed that $99.9 \%$ of Illumina reads and $99.2 \%$ of MinION reads were mapped to assembled genome. The genome was read with $575 x$ coverage for Illumina data and $454 x$ coverage for MinION data. Based on genome alignment $L$. fermentum FTDC8312 was found as closest related strain. The BLAST search identified the short contig as similar to bacterophage phiX174. In the genome 2120 coding sequences (CDS) were predicted. Among them 606 were annotated as "hypothetical" without known biological role. By using CAMPr3, ADAM and AMPA services 5 CDSs with highest summary prediction score were identified as coding for antimicrobial peptides. Further validation of their antimicrobial activity in vitro is required.
\end{abstract}

Key words - Lactobacilli, de novo assembly, antibacterial peptides.

\section{Introduction}

Lactobacilli, generally recognized as safe for humans, are characterized with high antagonistic properties. Besides some low-molecular agents, such as short-chain fatty acids, $\mathrm{H}_{2} \mathrm{O}_{2}$ and etc., Lactobacilli produce various antimicrobial peptides (AMPs) [1]. The resistance development to AMPs generally takes much longer period when compared to traditional antibiotics, that makes AMP promising antimicrobials considering rapid spread of drug-resistant pathogenic bacteria the screening for new antimicrobial agents. Among 40 strains of Lactobacilli isolated from the faeces of healthy humans, Lactobacillus fermentum HFD1 exhibited significant antimicrobial activity against various nosocomial pathogens [2]. From the culture liquid of $L$. fermentum HFD1 several antimicrobial compounds repressing growth of $P$. aureginosa were isolated by using reverse-phase chromatography. The chemical structure and nature of these antimicrobial metabolites remain to be identified and for this purpose the whole genome sequence is strictly required.

\section{Materials and methods}

DNA was extracted by phenol-chloform method. Sequencing was performed on Illumina MiSeq and ONT MinION instruments. Hybrid assemblers Unicycler v0.4.8beta [3] and SPAdes v3.13.0 [4], and long-read assembler Canu 1.8 [5] combined with short-read polishing with Pilon v. 1.23 [6] have been used. Genome has been annotated by using PROKKA 1.14.5 software [7]. Putative genes and genes clusters responsible for antimicrobial peptides synthesis were predicted by using ADAM [8], AMPA [9] and CAMPr3 [10] web-services.

\section{Results}

The combined sequencing on Illumina MiSeq and ONT MinION platforms allowed use advantages of both platforms. Illumina sequencing yielded 5 million filtered pair-end reads obtaining $1.2 \mathrm{Gbp}$ data. MinION generated 530 thousand reads with cumulative length of $1 \mathrm{Gbp}$ and maximum read length 53364. By comparing different strategies, Unicycler exhibited better performance whereas the assembly quality was the same for all assemblers. After assembly 2 circular contigs with respective sizes of $2101878 \mathrm{bp}$ and $5386 \mathrm{bp}$ has been obtained. The raw reads alignment with BWA and this post-assembly assessment showed that $99.9 \%$ of Illumina reads and $99.2 \%$ of MinION reads were mapped to assembled genome. Error rate was $8 \times 10^{-4}$ for Illumina and 0.1 for MinION. Finally, the genome was read with $575 \times$ coverage for Illumina data and $454 \times$ coverage for MinION data. Based on genome alignment with KmerFinder [11] L. fermentum FTDC8312 was found closest related strain. The BLAST search identified the short contig as similar to bacterophage phiX174.

Genome annotation was performed by using PROKKA software. In total 2120 coding sequences (CDS) were predicted. Among them 606 were annotated as "hypothetical" without known biological role. Data available in Genbank database by accession number CP050919. By using CAMPr3 (protocols SVM, RF, Ann DAC), ADAM and AMPA services 5 CDSs with highest summary prediction score were identified as coding for antimicrobial peptides with length from 56 to 133 amino acids. Further validation of their antimicrobial activity in vitro is required. 


\section{ACKNOWLEDGMENTS}

This research was funded by the Russian Foundation for Basic Research (grant number 17-00-00456) and by the subsidy allocated to Kazan Federal University for the state assignment in the sphere of scientific activities, project No. $13556.2019 / 13.1$

\section{REFERENCE}

[1] Gavrilova E. et al. (2019). Newly isolated lactic acid bacteria from silage targeting biofilms of foodborne pathogens during milk fermentation. BMC microbiology, 19(1), 248.

[2] Pavlova A. S. et al. (2020). Identification of Antimicrobial Peptides from Novel Lactobacillus fermentum Strain. The Protein Journal, 112 .

[3] Wick R. R., Judd L. M., Gorrie C. L., Holt K. E. (2017). Unicycler: resolving bacterial genome assemblies from short and long sequencing reads. PLoS computational biology, 13(6), e1005595.

[4] Bankevich A. (2012). SPAdes: a new genome assembly algorithm and its applications to single-cell sequencing. Journal of computational biology, 19(5), 455-477.
[5] Koren S., Walenz B. P., Berlin K., Miller J. R., Bergman N. H., Phillippy A. M. (2017). Canu: scalable and accurate long-read assembly via adaptive k-mer weighting and repeat separation. Genome research, 27(5), 722-736.

[6] Walker B. J. et al. (2014). Pilon: an integrated tool for comprehensive microbial variant detection and genome assembly improvement. PloS one, 9(11).

[7] Seemann T. (2014). Prokka: rapid prokaryotic genome annotation. Bioinformatics, 30(14), 2068-2069.

[8] Lee H. T., Lee C. C., Yang J. R., Lai J. Z., Chang K. Y. (2015). A largescale structural classification of antimicrobial peptides. BioMed research international, 2015.

[9] Torrent M., Nogués V. M., Boix E. (2009). A theoretical approach to spot active regions in antimicrobial proteins. BMC bioinformatics, 10(1), 373.

[10] Waghu F. H., Barai R. S., Gurung P., Idicula-Thomas S. (2016). CAMPR3: a database on sequences, structures and signatures of antimicrobial peptides. Nucleic acids research, 44(D1), D1094-D1097.

[11] Larsen, M. V. et al. (2014). Benchmarking of methods for genomic taxonomy. Journal of clinical microbiology, 52(5), 1529-1539. 